\title{
STUDY OF BL Lac VLBA DATA AT 8 AND 15 GHz IN A SUPER-RESOLUTION MODE
}

\author{
Claudio E. Tateyama ${ }^{1,2}$ \\ ${ }^{1}$ CRAAM-INPE/Escola de Engenharia-Universidade Presbiteriana Mackenzie, Rua da Consolação 896, 01302-907, São Paulo, SP, Brazil \\ 2 CEA-Instituto Nacional de Pesquisas Espaciais-INPE, Av. dos Astronautas, 1758, 12201-970, São José dos Campos, SP, Brazil \\ Received 2009 June 8; accepted 2009 September 28; published 2009 October 15
}

\begin{abstract}
In this work, we show Very Long Baseline Array data at $8 \mathrm{GHz}$ (RRFID) and $15 \mathrm{GHz}$ (MOJAVE) of BL Lac from 1995 to 2007 by examining the structure in the maps given by the CLEAN-point components (represented by a restoring beam of 0.1 mas). The CLEAN-point maps show a well-ordered train of individual points in the inner core jet. The result shows a narrow elongated stationary component at about 1.5 mas from the core which was interpreted as superposition of trailing components. The inner core-jet structure shows ballistic motion and a precessing ejection nozzle period of 26 years.
\end{abstract}

Key words: BL Lacertae objects: individual (BL Lac) - galaxies: jets - radio continuum: galaxies - techniques: interferometric

\section{INTRODUCTION}

BL Lac is a remarkable variable radio source in the nucleus of an elliptical galaxy at a redshift of 0.069 (Miller et al. 1978). Very Long Baseline Interferometry (VLBI) observations of the parsec-scale structure of BL Lac reveal a bright compact radio core and a wiggling structure extending to the south, e.g., Mutel et al. (1990), Tateyama et al. (1998), Denn et al. (2000), Stirling et al. (2003), and Jorstad et al. (2005). High-resolution Very Long Baseline Array (VLBA) maps at $43 \mathrm{GHz}$ of inner core jet (about 1.5 mas in length) obtained by Stirling et al. (2003) and Jorstad et al. (2005) indicate ballistic motion for superluminal components emerging from the core at different ejection angles. A precessing nozzle period of 2.3 years was suggested by Stirling et al. (2003). Stationary component is a well-known feature in the context of a superluminal source. The obvious connections of such static features are bent jet, jet interaction with intergalactic cloud or reconfinement shock. The first report of the stationary component in BL Lac appeared in the work done by Mutel et al. (1990) denominated S4 which lasted for a few months. Stirling et al. (2003) reported a faint component with constant radial separation from the core of 1.5 mas. This work presents a study of the source structure and sidelobe features using VLBA observations at 8 and $15 \mathrm{GHz}$ in a super-resolution mode.

\section{DATA AND IMAGING PROCEDURE}

We have used visibility VLBA data at $8 \mathrm{GHz}$ from the Radio Reference Frame Image Database (RRFID) of the US Naval Observatory (USNO) and at $15 \mathrm{GHz}$ were obtained from MOJAVE (Monitoring of jets in active galactic nuclei with VLBA experiments) program. The data include a set of maps containing adequate time sampling observations in order to recognize features of the source in adjacent maps. In Table 1, we list the VLBA data used in this work. The columns of the table are 1: epoch, 2: frequency of the observation, 3: peak brightness, 4: synthesized beam size, and 5: position angle (P.A.) of the beam. The images were obtained using Difmap Package (Shephard 1997) and processed in an automate mode using mapping script of Taylor and Shephard. The restoring beam was 0.1 mas. The pixel size of the images was 0.05 mas.
The CLEAN algorithm produces a set of point components which convolved with the CLEAN beam and superposed on the residual map generates the CLEAN map. In the case of super resolved map, the convolution is made with much smaller beam. The noise level of the map is estimated from the visibility and gridding weights. By convolving with a smaller beam, we give higher weight to visibilities at the edges of the $(u, v)$ plane, which modifies the noise of the map. In effect, the location of individual point components in the inner core jet has more weight, which gives more weight to the sharpened structure.

\section{RESULTS AND DISCUSSION}

Figure 1 presents the VLBA maps of BL Lac from 1995 to 2007 at $8 \mathrm{GHz}$ and $15 \mathrm{GHz}$. Distinct features are readily recognizable in the maps. The core is the brightest component represented by strong CLEAN-point with two subcomponents located symmetrically on opposite sides of the central component. The direction of this three-component core is assumed to be in the ejection angle of the nozzle. By the "core", we mean the bright unresolved feature typically found at the end of the so-called core-jet sources. Compact components in the jet produce single CLEAN-point knots, and extended components show rings and radial lines that can be followed in contiguous maps.

The CLEAN-point maps at $8 \mathrm{GHz}$ and $15 \mathrm{GHz}$ show similar features $(8 \mathrm{GHz}$ data include geodetic VLBI antennas which give a resolution closer to the $15 \mathrm{GHz}$ data). The contour lines of the maps are shown only for the strongest components (contour lines above $1 \%$ of the peak flux). The inner core-jet structure shows a train of CLEAN-point components indicating an ejection angle predominantly southward with small inclination to the west from 1995 to 2007 except in 2005 September through 2006 April when the ejection angle appears on the east side.

S8, S9, and S10 are well-known superluminal components observed during 1995 through 2000 in several VLBI and VLBA observations (Tateyama et al. 1998; Denn et al. 2000; Stirling et al. 2003). Here, they are shown in the description of CLEANpoint maps. S10 exhibits circular shape, ring, and radial features as expected for a discreet (extended) component and can be seen moving down smoothly along the length of the jet from 1997 August to 1999 January. S10 is brighter than the core in February 
Table 1

VLBA Data at $8 \mathrm{GHz}$ (RRFID) and $15 \mathrm{GHz}$ (MOJAVE) Used in this Work

\begin{tabular}{|c|c|c|c|c|}
\hline Epoch & $\begin{array}{c}\text { Frequency } \\
(\mathrm{GHz})\end{array}$ & $\begin{array}{c}I_{\text {peak }} \\
\left(\mathrm{Jy} \mathrm{beam}^{-1}\right)\end{array}$ & $\begin{array}{c}\text { Beam } \\
(\text { mas } \times \text { mas })\end{array}$ & $\begin{array}{c}\text { P.A. } \\
\left({ }^{\circ}\right)\end{array}$ \\
\hline 1995 Apr 9 & 15 & 1.8 & $0.8 \times 0.5$ & 5 \\
\hline 1995 Dec 16 & 15 & 2.1 & $0.9 \times 0.5$ & 2 \\
\hline 1996 May 16 & 15 & 2.7 & $0.9 \times 0.5$ & -3 \\
\hline 1996 Oct 27 & 15 & 1.8 & $0.9 \times 0.5$ & -1 \\
\hline 1997 Jan 30 & 8 & 1.3 & $1.0 \times 0.7$ & -9 \\
\hline 1997 Mar 10 & 15 & 1.5 & $1.0 \times 0.6$ & 8 \\
\hline 1997 Mar 31 & 8 & 1.1 & $1.0 \times 0.8$ & -1 \\
\hline 1997 Мay 19 & 8 & 1.6 & $1.1 \times 0.9$ & -16 \\
\hline 1997 Jul 24 & 8 & 1.2 & $1.1 \times 0.8$ & -8 \\
\hline 1997 Aug 28 & 15 & 1.7 & $0.9 \times 0.6$ & 12 \\
\hline 1997 Sep 8 & 8 & 1.2 & $1.1 \times 0.8$ & -7 \\
\hline 1997 Dec 17 & 8 & 1.7 & $0.9 \times 0.8$ & -11 \\
\hline 1998 Feb 9 & 8 & 1.0 & $0.9 \times 0.7$ & -24 \\
\hline 1998 Mar 7 & 15 & 1.1 & $1.0 \times 0.5$ & 9 \\
\hline 1998 Apr 15 & 8 & 1.3 & $1.1 \times 0.7$ & -11 \\
\hline 1998 Jun 25 & 8 & 1.0 & $0.8 \times 0.5$ & -13 \\
\hline 1998 Aug 10 & 8 & 1.6 & $1.0 \times 0.8$ & -18 \\
\hline 1998 Dec 21 & 8 & 1.3 & $1.0 \times 0.7$ & -19 \\
\hline 1999 Jan 2 & 15 & 1.6 & $0.8 \times 0.6$ & -16 \\
\hline 1999 Nov 6 & 15 & 2.3 & $1.3 \times 0.6$ & -10 \\
\hline 2000 Dec 28 & 15 & 1.8 & $1.0 \times 0.6$ & -21 \\
\hline 2001 Dec 22 & 15 & 1.5 & $1.1 \times 0.6$ & 0 \\
\hline 2002 Jan 16 & 8 & 1.1 & $1.0 \times 0.7$ & -21 \\
\hline 2002 Jun 15 & 15 & 1.3 & $0.8 \times 0.6$ & -17 \\
\hline 2003 Feb 6 & 15 & 1.0 & $0.9 \times 0.6$ & -1 \\
\hline 2003 Aug 28 & 15 & 2.4 & $0.8 \times 0.5$ & 1 \\
\hline 2004 Jun 11 & 15 & 2.1 & $0.8 \times 0.6$ & -7 \\
\hline 2004 Oct 18 & 15 & 2.4 & $0.8 \times 0.7$ & -35 \\
\hline 2005 Sep 16 & 15 & 3.4 & $0.8 \times 0.6$ & -11 \\
\hline 2005 Sep 23 & 15 & 3.5 & $0.7 \times 0.6$ & 10 \\
\hline 2006 Apr 5 & 15 & 3.0 & $0.8 \times 0.6$ & -9 \\
\hline 2006 Nov 10 & 15 & 1.4 & $0.8 \times 0.6$ & -10 \\
\hline 2007 Feb 5 & 15 & 2.2 & $1.0 \times 0.6$ & -22 \\
\hline 2007 Mar 28 & 15 & 2.8 & $0.8 \times 0.6$ & -8 \\
\hline 2007 Jun 10 & 15 & 2.7 & $0.8 \times 0.6$ & -21 \\
\hline
\end{tabular}

and March of 1998, and located at about 2 mas from the core. S8 can be recognized in the maps from 1995 April to 1996 October. S9 is seen from 1996 October to 1997 September. S9 and S10 appear on the same maps in August and September of 1997. The comparison of CLEAN-point maps from 1998 February to 2000 December with high-resolution maps at 43 $\mathrm{GHz}$ of (Stirling et al. 2003; Jorstad et al. 2005) shows striking similarity of the structure in the smallest details. Figure 2 shows the comparison of maps of VLBA observations in 1998 at 8, 15, and $43 \mathrm{GHz}$

The super-resolution maps show a notable change of pattern in the maps starting from about the year 2000. The well-known pattern of superluminal components moving smoothly down the jet (components S9 and especially S10; maps on the upper side of Figure 1) is no longer seen on the maps of later epochs (lower side of Figure 1). The sidelobe "arcs" (lower side of Figure 1) become prominent and a pair of CLEAN-point components stands out at 1.1 and 1.6 mas on the later epoch maps.

\subsection{CLEAN Instability and Dirty Beam}

Figure 3(a) shows the sequence of three maps (February, March, and June of 2007) of Figure 1. The map of 1997 February shows bright points along a straight radial line near the core, in 2007 March the bright points appear slightly curved to the east. The CLEAN-point components exhibit no radial motion during the period between February and June, however, source motion (superluminal motion) can be deduced from the transfer of bright CLEAN-point from a given "arc" to an outer "arc."

The superposition of maps in Figure 3(b) (February, March, and June of 2007) and especially the superposition of four years data in Figure 4 (2003 February to 2007 June, see Table 1) show that the distribution of CLEAN-point components appears sharply located in a series of (equally spaced) fixed "arcs." An extended feature on the east side of the source appears remarkably in the superposition. This feature is the outer trajectory of the jet components (about 3 mas from the core) which has an intrinsic (nonballistic) bent to the east. Figure 5 shows the feature in a larger scale map. The eastern feature extends further to the south and gets confused with large-scale sidelobe "arcs" drawing a well-defined spiral figure.

\subsubsection{Simulation}

Most of the features of CLEAN-point images were examined by carrying out a simple simulation of VLBA image using the task UVCON along with the IMMOD task in AIPS. The simulated data were imaged using the Difmap Package. CLEAN instability produces image modulation of spatial frequencies corresponding to unsampled parts of the $(u, v)$ plane (Cornwell 1983). The super resolved data (CLEAN-point maps) tend to amplify the appearance of modulation. In the work done by Lister et al. (2001), these modulations are shown as a sinusoidal ripple running along the length of the jet. In this way, the "arcs" in Figure 4 would be related with the undersampling of outer region of the $(u, v)$ plane and in Figure 5 with the undersampling of the inner region of the $(u, v)$ plane. The simulated VLBA image (noise free) in Figure 6 shows clearly that the large-scale "arcs" are concentric features in the $(u, v)$ plane, in contrast with the extension of the eastern feature in Figure 5 suggesting a spiral structure centered $\sim 7$ mas south of the core. For instance, the angular resolution, $\theta_{\mathrm{HPBW}}$, (half-power beamwidth) of a VLBA map is given by

$$
\theta_{\mathrm{HPBW}} \sim 2063 \times \frac{\lambda^{\mathrm{cm}}}{B_{\max }^{\mathrm{km}}} \text { mas, }
$$

where $\lambda^{\mathrm{cm}}$ is the wavelength in $\mathrm{cm}$ and $B_{\max }^{\mathrm{km}}$ is the maximum baseline in $\mathrm{km}$. The shortest baseline (Pie Town-Los Alamos) of $237 \mathrm{~km}$ at $15 \mathrm{GHz}$ gives a size of 17 mas and the longest baseline (Mauna Kea-Saint Croix) of $8612 \mathrm{~km}$ gives a size of 0.5 mas. These values are about the outer and inner spacing of the "arcs" in the maps.

In particular, the CLEAN algorithm locates the maximum in the broad feature and removes a point-source component. There is a tendency for the component-subtraction points to be located at an interval equal to the spacing of the first sidelobe of the originally synthesized beam (Clark 1982). Here, the component subtraction related to double CLEAN-points at about 1.1 and 1.6 mas locates the points at interval equal to the spacing of holes in the $(u, v)$ plane.

Besides examining general aspects of the super resolved maps, we have also done simulation of the observed data at $15 \mathrm{GHz}$ (February, March, and June of 2007) to analyze in more detail the feature related with the stationary component (the strongest component in the jet). The initial model source was based on the Gaussian components obtained from MODELFITing (Difmap Package) the CLEAN observed image. The final model source was obtained through the best match to the CLEAN observed image as well as CLEAN-point image. 

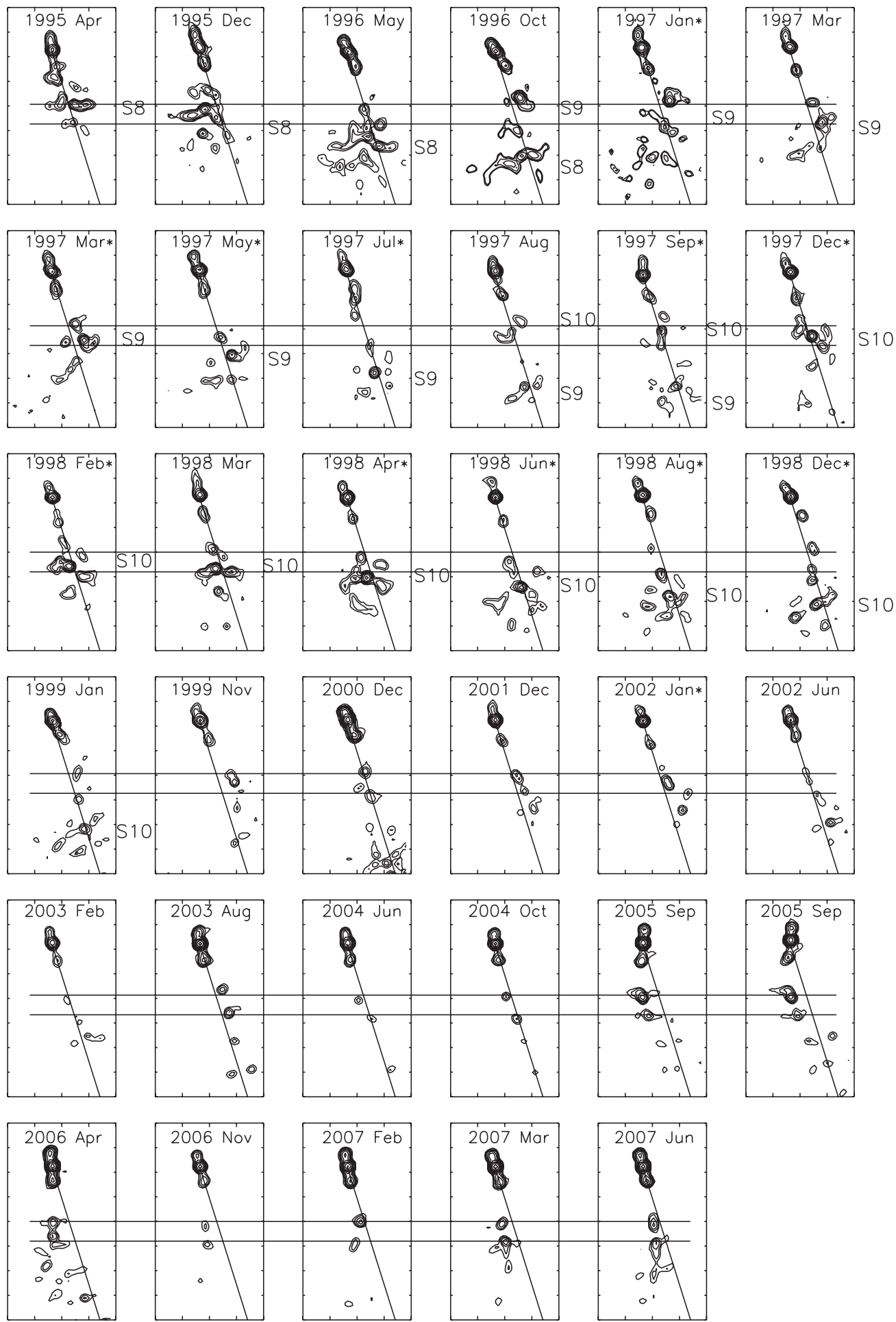

Figure 1. 8 and $15 \mathrm{GHz}$ VLBA images of BL Lac. Contour levels are 1, 2, 4, 8, 16, 32, 64, 128, 256, and $512 \times 0.011 \mathrm{Jy}^{\text {beam }}{ }^{-1}$. The asterisk in the date of maps indicates data at $8 \mathrm{GHz}$. The interval of tick marks is 0.5 mas. The diagonal line is a reference to guide the eye through variation of ejection angle and the double horizontal lines serve as a reference to the static points at radial distance of 1.1 and 1.6 mas. The restored circular beam is 0.1 mas. The superluminal components S8, $\mathrm{S} 9$, and S10 are indicated in the figure.

Figure 7 shows the source model at $15 \mathrm{GHz}$ of 2007 February, March, and June.

The stationary component revealed a narrow and elongated structure of $0.28 \times 0.76$ mas along the length of the jet in February and March which became a little larger in June $(0.28 \times 1.05$ mas). The P.A. does not change but the radial position increases from 1.3 mas in February to 1.6 mas in March and June. The model source (stationary component) breaks into two individual CLEAN-point components at 1.1 and 1.6 mas from the core in the super resolved maps as shown in Figure 8. The result shows, interestingly, that a stationary component coincides with the location of the double CLEANpoint components (1.1 and 1.6 mas from the core).

Stationary component is a well-known feature in BL Lac. S4 was a stationary component at 1.35 mas from the core which lasted for few months Mutel et al. (1990). A faint component 


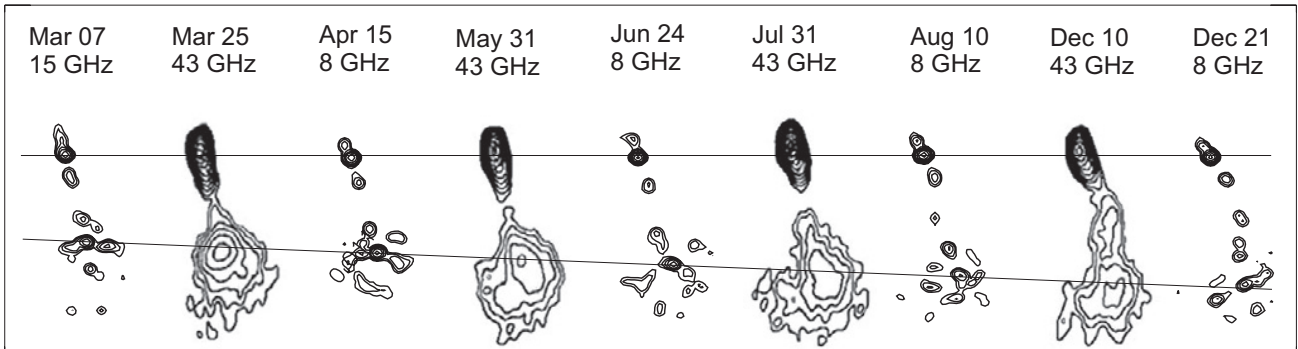

Figure 2. Comparison of CLEAN-point maps at 8 and $15 \mathrm{GHz}$ with $43 \mathrm{GHz}$ maps. The $43 \mathrm{GHz}$ is an adaptation of Figure 13 of Jorstad et al. (2005)

BL Lac

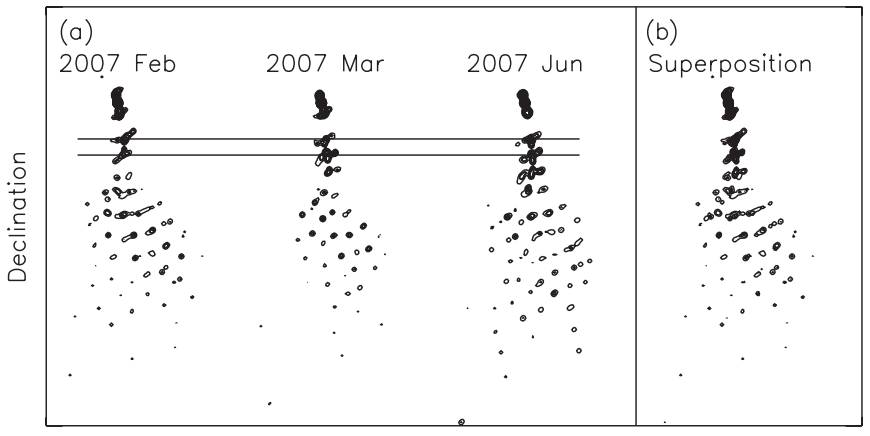

Right Ascension

Figure 3. (a) Maps of February, March, and June of 2007 from Figure 1. (b) Superposition of maps. Contour levels are $0.13,0.25,0.5,1,2,4,8,16,32,64$, 128 , and $256 \times 0.011 \mathrm{Jy}$ beam $^{-1}$.

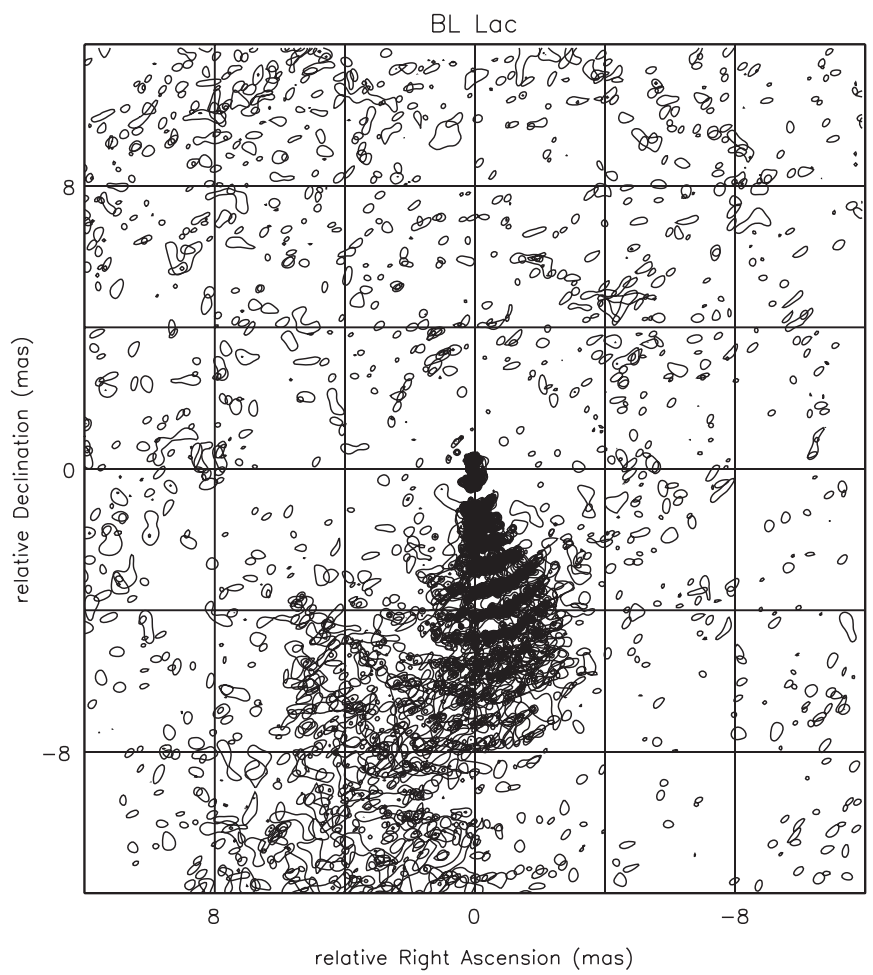

Figure 4. Superposition of CLEAN-point components from 2003 to 2007 of BL Lac. Contour levels are 0.06, 0.13, 0.25, 0.5, 1, 2, 4, 8, 16, 32, 64, 128, and $256 \times 0.011 \mathrm{Jy}^{\mathrm{beam}}{ }^{-1}$.

at a constant radial separation at $\sim 1.5$ mas from the core has been reported by Stirling et al. (2003). A lack of emission between the core and the jet component in the inner 1 mas from the core, the low emission region between 0.7 and 1 mas

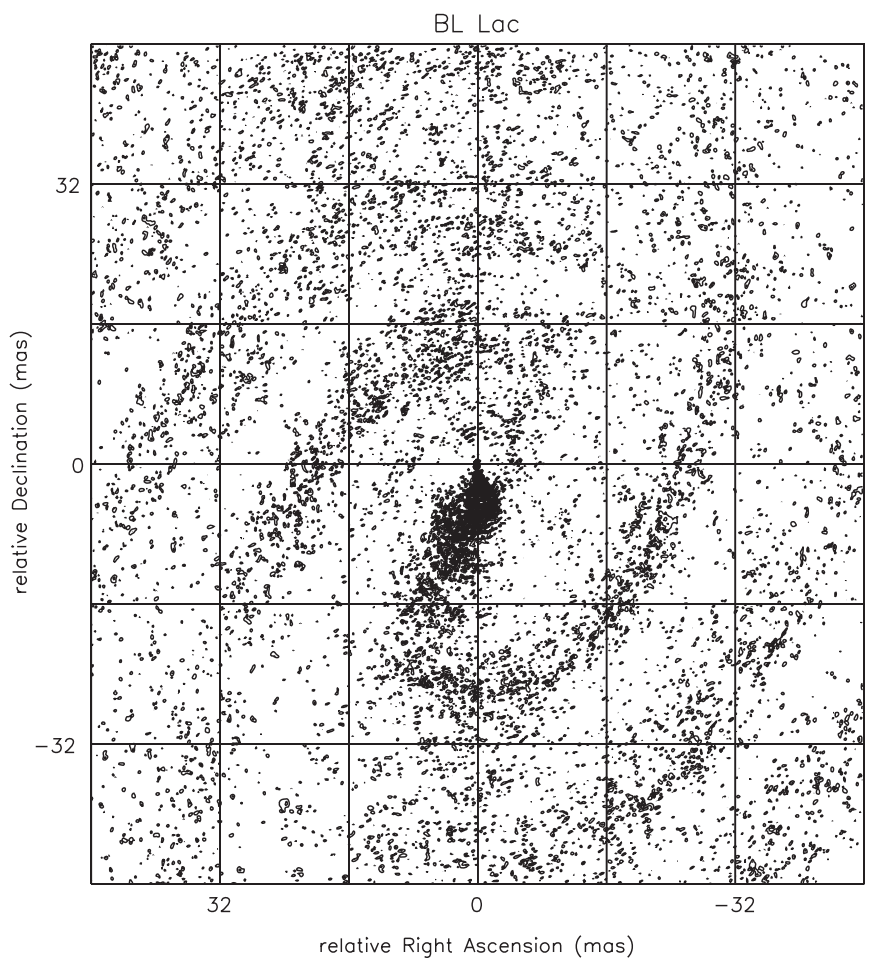

Figure 5. Large-scale (100 mas) superposition of CLEAN-point components from 2003 to 2007 of BL Lac. Contour levels are 0.05, 0.06, 0.13, 0.25, 0.5, 1, $2,4,8,16,32,64,128$, and $256 \times 0.011 \mathrm{Jy} \mathrm{beam}^{-1}$.

in the profile jet ridgeline at P.A. $195^{\circ}$ at $43 \mathrm{GHz}$ (Figure 2 of Bach et al. 2006) from 1997 to 2003 lead to a especial feature around the position of the stationary component. In fact (1) the jump of the bright CLEAN-point component in successive outer CLEAN-point components (see Figure 3), (2) the increase of the radial position and the size of the model component of the stationary component between February and June in the simulation data, and also, (3) inspection of the maps (2003 through 2007) of Figure 1 showing that the P.A. of double CLEAN-point is not static provide firm evidence of the variation of structure of the stationary component. It suggests a kind of a static window in the jet with moving components in it. Namely, such constant radial feature can be interpreted as a train of short lived moving components. New components appear at about 1.1 mas and join to earlier components to form a narrow train of individual point components of about 1 mas in length. The blend of such moving point sources would appear observationally as a stationary component.

We examined the core structural P.A. and the major axis of the stationary component in the maps. In particular, a trailing component can be formed in the wake of strong component 


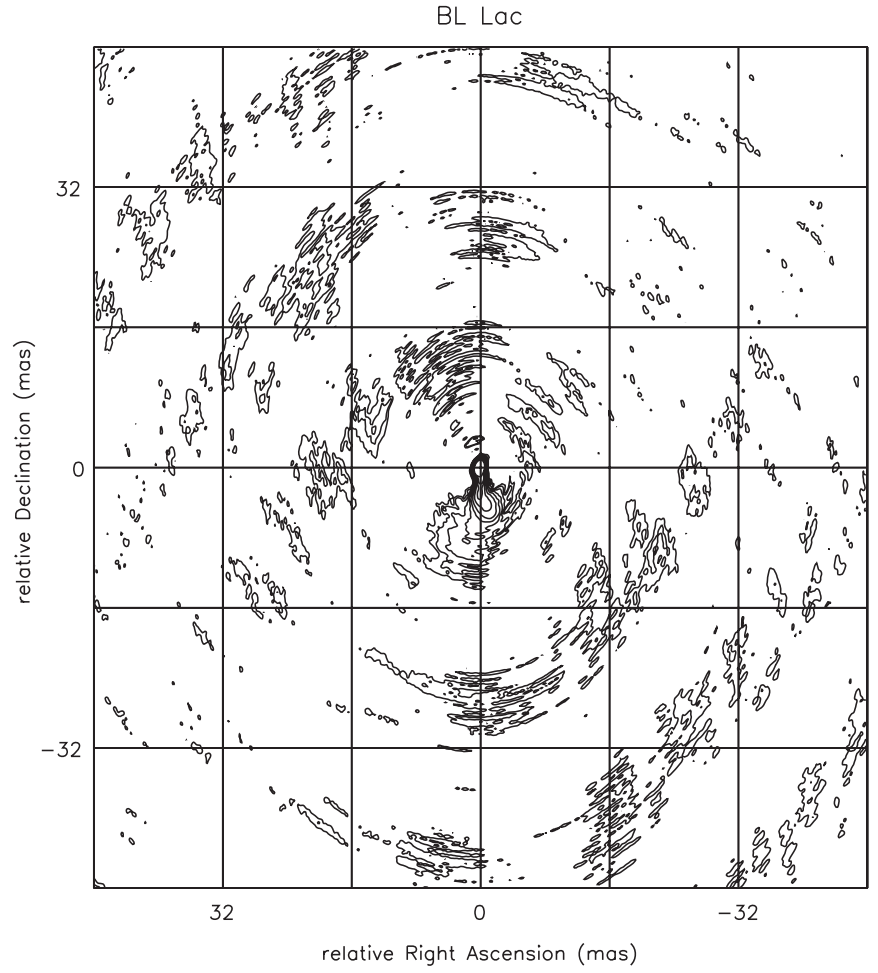

Figure 6. CLEAN beam simulated VLBA map at $15 \mathrm{GHz}$. Contour levels are $0.03,0.06,0.13,0.25,0.5,1,2,4,8$, and $16 \times 0.011 \mathrm{Jy}^{\text {beam }}{ }^{-1}$.

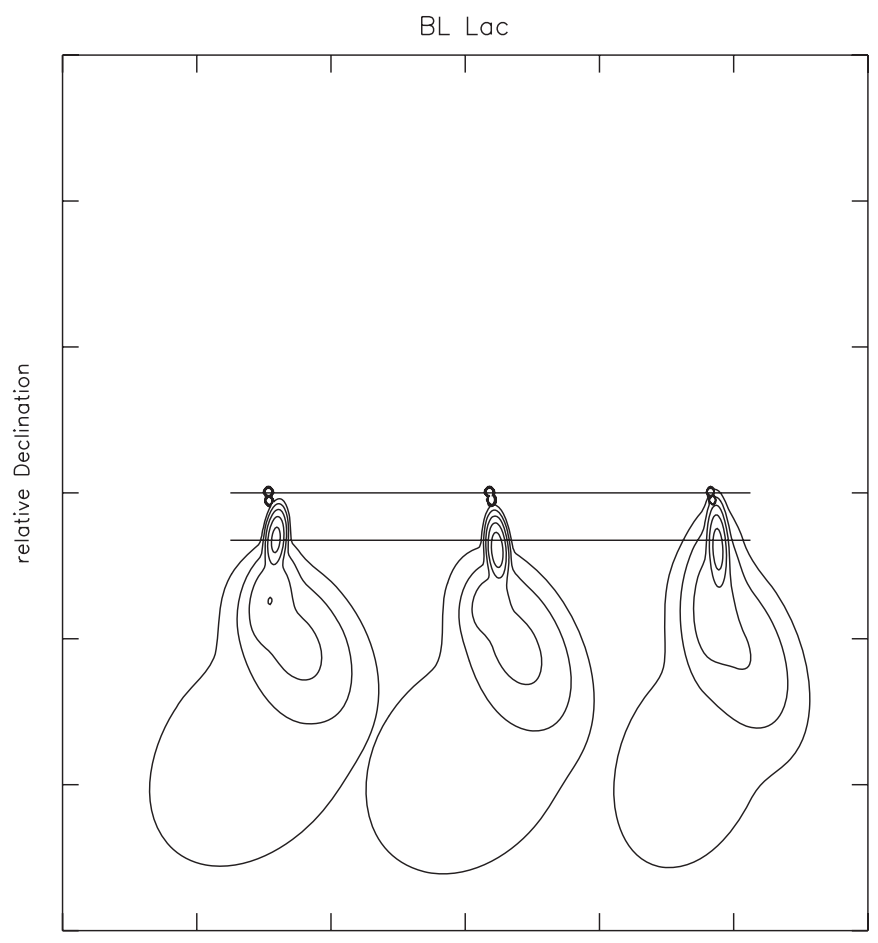

relative Right Ascension

Figure 7. Model source at $15 \mathrm{GHz}$ of February, March, and June of 2007. Contour levels are $0.13,0.5,2,8$, and $32 \times 0.011 \mathrm{Jy}^{\text {beam }}{ }^{-1}$. The tick interval is 4 mas.

(Agudo et al. 2001). Although the leading knot (knots) is (are) not recognizable in BL Lac, we can assume that the stationary component would be composed by a superposition of many trailing components. The change of direction of nozzle would produce a misalignment between the major-axis P.A. of the

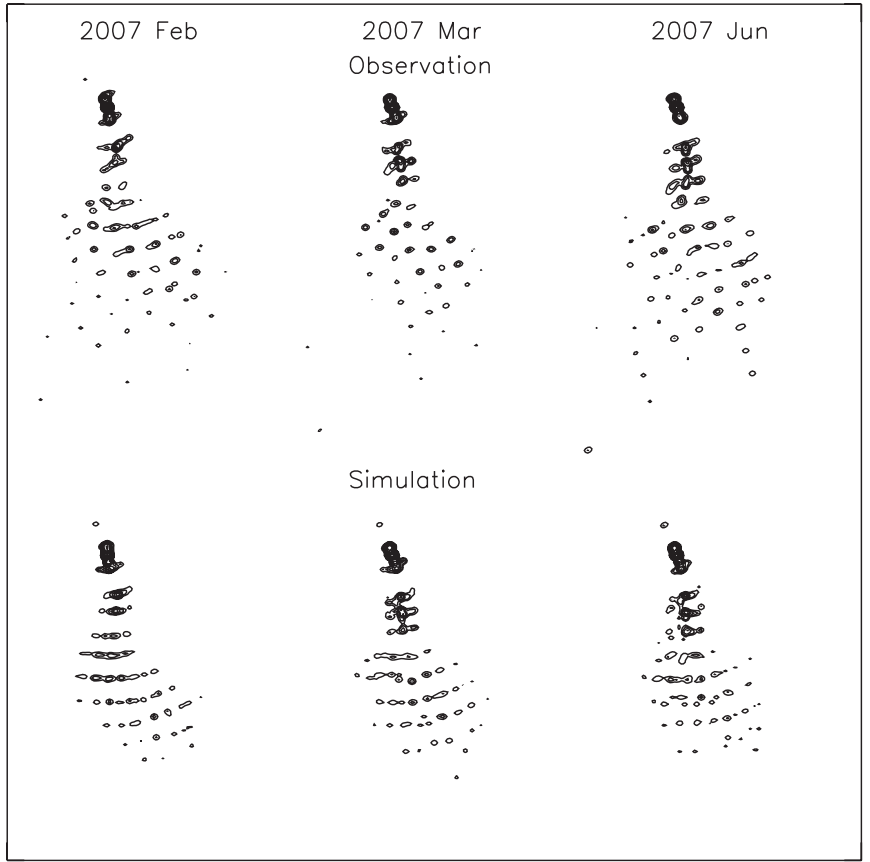

Figure 8. Simulated VLBA CLEAN-point maps (below) compared with the observed CLEAN-point maps (above) at $15 \mathrm{GHz}$ of February, March, and June of 2007. Contour levels are $0.13,0.25,0.5,1,2,4,8,16,32,64,128$, and $256 \times 0.011 \mathrm{Jy}$ beam $^{-1}$.

stationary component relative to the structural P.A. of the core. This is clearly shown in the model source of 2007 February (Figure 7), an aspect that gives a firm evidence for ballistic motion of components. This is also in agreement with rectilinear trajectory of individual components observed at $43 \mathrm{GHz}$ by Stirling et al. (2003) in the inner core jet.

Jorstad et al. (2005) pointed out that the change of the direction of nozzle between ejections is important, since the second major disturbance passing through a trailing shock would destroy it. By the way, the stationary component of BL Lac would not be constituted of subluminal trailing components, since our data (Figure 1) show that P.A. of stationary component changes with time, suggesting that trailing components are of relative short duration, or bright only within the window of the stationary component.

As to the change of pattern of CLEAN-point maps, a simple simulation shows that, the passage of weak jet components along the holes in the $(u, v)$ plane would produce the appearance of well-defined sidelobe "arcs" at fixed radial distances (the shapes of the "arcs" are unaffected by the passage of the source components). The sidelobe would be enveloped by the structure of the source (size of the component). On the other side, the pattern seen on the upper side of Figure 1 would be reproduced by strong components moving down the jet deforming their own sidelobes around the jet component. S10 is the best example of such feature which can be seen moving smoothly down the jet exhibiting rings and radial lines. Hence, the intensity of the components in the jet relative to the sidelobes produces the distinct patterns shown in the maps of Figure 1.

In summary, the super-resolution map shows robust description of source structure, with the longitudinal direction of the components appearing as one of special features of CLEANpoint map to examine the change of direction of the structural P.A. of components near the core (the core itself and stationary component). Although artifacts due to Clean instability are well-known features in VLBA images, this work through 

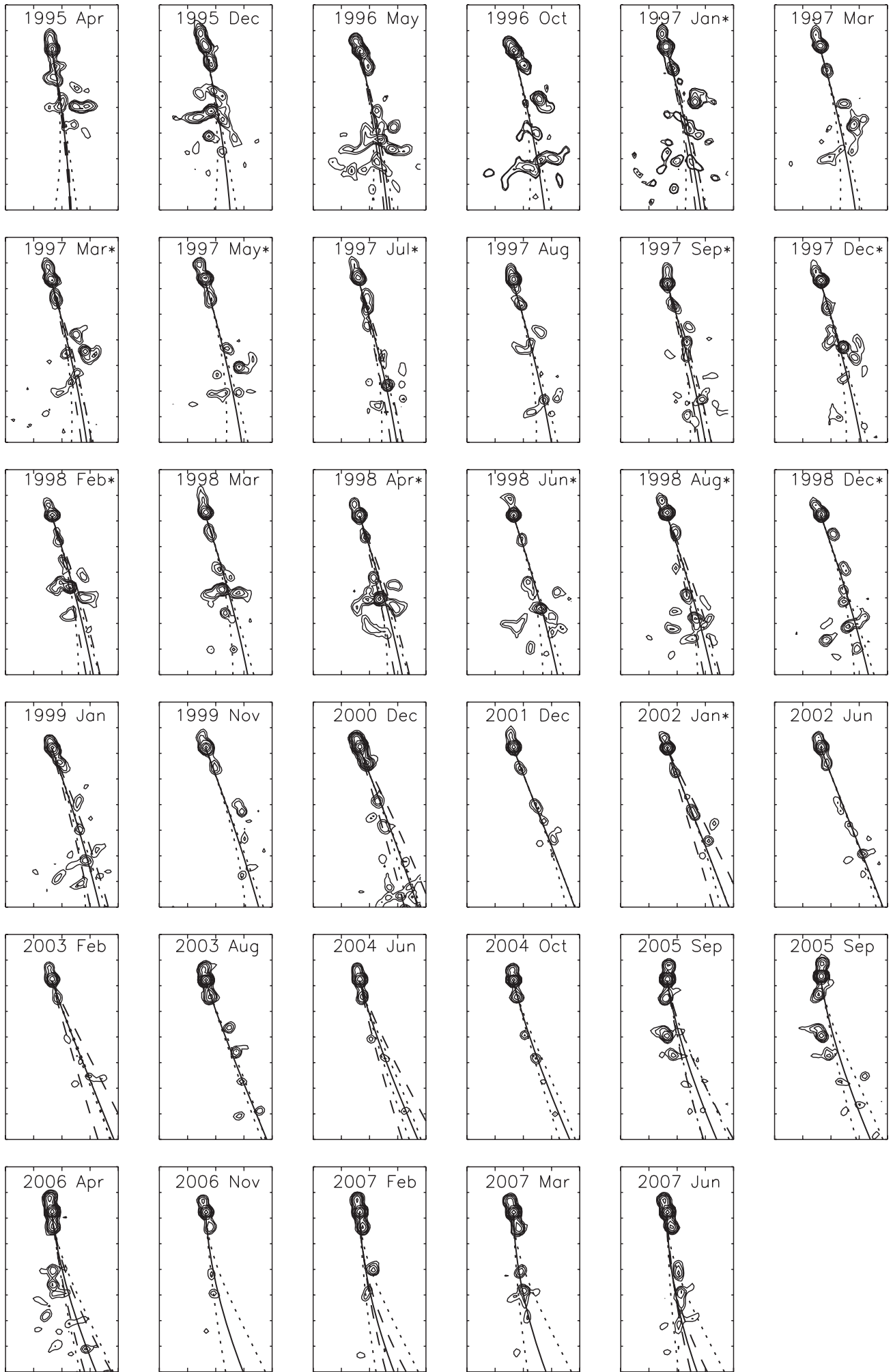

Figure 9. Same as Figure 1. The ridgelines represent the precessing ballistic motion. The solid line represents the best model fit, the dotted line represents the speed $\beta$ for 0.989 and 0.999 , and the dashed line, on alternate maps, represents the opening angle $\Omega$ for 0.4 and 0.8 .

superposition of CLEAN-point maps highlights their presence in the maps allowing further examination of the source structure. The double CLEAN-point component is a persistent feature that appears at about 1.5 mas and related to the stationary component. It is not a static region in the jet as we would expect from a bent jet, the motion can be seen in the source models in Figure 7, or even more clearly described by the evolution of CLEAN-point features in the maps of Figure 3 or Figure 8. The stationary component exhibits an elongated structure. The change of direction of the nozzle ejection is an important point on the appearance of the stationary component as a superposition of trailing components. 


\subsection{Precessing Ballistic Motion}

The track along the circle of $(u, v)$ plane especially at the edges of $(u, v)$ plane provides denser data sample relative to the direction of the core, or even the inner jet, compared to radial "tracks." Hence, super resolved maps would show sharper visual details relative to the direction of inner core jet. The ridgeline of the core-jet ( $\sim 2$ mas) structure in Figure 1 shows a well-ordered train of points presumably describing a ballistic trajectory. The direction of the ejection is predominantly southward with small inclination to the west from 1995 to 2007 except in 2005 September to 2006 April when it appears on the east side. We consider the ridgeline of the core jet ("stationary component" included) with a size of about 1.5 mas. If we ignore the rapid oscillation (2.3 years proposed by Stirling et al. 2003) and also the outer jet ( $\geqslant 3$ mas) which clearly exhibits nonballistic trajectory, the overall motion (precession) of ejection angle with time suggests a rather long period. Assuming a precessing ballistic model (Hjellming \& Johnston 1981; Tateyama \& Kingham 2004), we start the search of the parameters to fit the observed ridgeline by using a reasonable combination of the parameters. We note that for the length of the ridgeline considered here, the aperture angle of cone $\Omega \propto$ viewing angle. Thus, the same ridgeline shape can be reproduced just multiplying the parameters $\Omega$ and viewing angle by a factor. The constraint of $\Omega$-viewing angle parameter (factor) and hence estimation of jet velocity are obtained from the observed proper motion (Equations (2) and (3)). The best fit to the change of the ejection angle in the maps is shown in Figure 9.

On the basis of visual inspection the agreement between data and model is good. The position of bright knots in 1996 October, 1997 January, 2005 September, and 2006 April which diverges from the ridgeline of the jet model can be associated with shorter term (or non-periodic) motion of a longer period of precession. S9 in 1996 October shows a pronounced change in the P.A. compared with the P.A. of the earlier knots. This feature is also present in the work of Tateyama et al. (1998) and Denn et al. (2000). The trajectory of S9 can be described as showing initially a strong curvature from 1996 October to 1997 July but from 1997 July to 1997 September S9 is seen having a rectilinear trajectory. An alternative description of S9 can be related to its emissivity and structure. The initial bright feature of S9 in 1996 October and 1997 January may represent a brighter section (portion of a ring) of a larger component (ring) centered on the ridgeline of the precessing jet. As the component moves down the jet the full ring feature with bright knot in the center shows up. The component is all the time in ballistic motion. This explanation reinforces the jet model.

The precessing ballistic motion has a period, $T$, of 26 years. Particularly, the 2.3 oscillation of Stirling et al. (2003) is too short. The correlated periodicity of historical radio and optical light curves of eight years obtained by Villata et al. (2004) would easily show up in the features of the maps. The period of 14 years in optical reported by Fan et al. (1998) is still inadequate for description of ejection angle through the maps as it would require large cone aperture and small jet velocity as seen below. The morphological aspect of the ridgeline suggests a rather extreme jet parameters for BL Lac with an aperture angle of precessing cone, $\Omega$, of 0.6 , its axis forms an angle, $\phi$, of 2.4 with the line of sight, and a P.A. of $180^{\circ}$ in the plane of the sky. The jet velocity is $0.996 c$.

The error estimate was obtained by varying each parameter while holding all others fixed. The standard deviation $\sigma$ of the best model is 0.075 mas. For a $\Omega$ of $0.4-0.8$ (see Figure 9) the standard deviation is $1.47 \sigma-1.64 \sigma$; for a $\phi$ of $1.8-3.6$ the standard deviation is $1.68 \sigma-1.44 \sigma$; for a P.A. of $176^{\circ}-184^{\circ}$ the standard deviation is $1.72 \sigma-1.44 \sigma$; and for a $\beta$ of $0.989-0.999$ the standard deviation is $1.31 \sigma-1.15 \sigma$. In the case of speed $\beta$, the standard deviation is relatively smaller because the trajectories "converge" toward the best model as shown in Figure 9. The period presents similar results with a $T$ of 22-30 years giving the standard deviation of $1.32 \sigma-1.33 \sigma$.

The apparent velocity $\beta_{\text {app }}$ is given by

$$
\beta_{\text {app }}=\frac{\beta \sin \theta}{1-\beta \cos \theta},
$$

where $\theta$ is the viewing angle. For a jet speed $\beta$ of 0.996 , the maximum apparent velocity $\beta_{\text {app }}^{\max }=\left(1 / \beta^{2}-1\right)^{-1 / 2}=11.2$. The relation between apparent jet velocity $\beta_{\text {app }}$ and proper motion $\mu$ in mas $_{\mathrm{yr}^{-1}}$ is

$$
\beta_{\text {app }}=3.11 \frac{\mu}{h_{0}}=4.4 \mu \text {. }
$$

For $z=0.069$, Hubble constant of $71 \mathrm{~km} \mathrm{~s}^{-1} \mathrm{Mpc}^{-1}$ and deceleration parameter $q_{0}=0.5$, the proper motion $\mu$ at maximum apparent velocity is 2.5 mas $^{\mathrm{yr}^{-1}}$ which is about the highest proper motion $\left(\sim 1.9\right.$ mas $\left.\mathrm{yr}^{-1}\right)$ seen in BL Lac. It is not unreasonably high if the beaming is restricted only to the generation of the precessing jet itself (precessing motion of 26 years). The observed (lower and variable) proper motions and higher wobbling of the inner jet in BL Lac must combine a motion of long-term precessing jet with a short-term oscillation, for example, an oscillation of about 2.3 years as proposed by Stirling et al. (2003), and probably a non-periodic motion.

\section{CONCLUSION}

In this work, we show VLBA data at $8 \mathrm{GHz}$ (RRFID) and $15 \mathrm{GHz}$ (MOJAVE) of BL Lac from 1995 to 2007 by examining the structure in the maps given by the CLEAN-point components. The CLEAN-point maps show a well-ordered train of individual points in the inner core jet. The result shows a narrow elongated stationary component at about 1.5 mas from the core which was interpreted as superposition of trailing components. The configuration given by the structural P.A. of the core and major axis of stationary component shows strong support for ballistic motion. The change of the P.A. of inner core jet (core plus stationary component) was fitted to the ballistic components being ejected by a precessing nozzle of a period of 26 years.

I thank the referees for useful suggestions and comments. I thank A.V.R. Silva for reading the paper and V. M. S. Pereira for helping with Figure 2. This research made use of the United States Naval Observatory (USNO) Radio Reference Frame Image Database (RRFID). This research also used data from MOJAVE (Lister \& Homan 2005) and the $2 \mathrm{~cm}$ Survey (Kellermann et al. 2004) programs.

\section{REFERENCES}

Agudo, I., Gómez, J. L., Martí, J. M., Ibáñez, J. M., Marscher, A. P., Alberdi, A., Aloy, M. A., \& Hardee, P. E. 2001, ApJ, 549, L183

Bach, U., et al. 2006, A\&A, 456, 105

Clark, B. G. 1982, in Proc. NRAO Workshop 5, Large Field Mapping in Synthesis Mapping, ed. A. R. Thompson \& L. R. DAddario (Green Bank, WV: National Radio Astronomy Observatory) 
Cornwell, T. J. 1983, A\&A, 121, 281

Denn, G. R., Mutel, R. L., \& Marscher, A. P. 2000, ApJS, 129, 61

Fan, J. H., Xie, G. Z., Pecontal, E., Pecontal, A., \& Copin, Y. 1998, ApJ, 507, 173

Hjellming, R., \& Johnston, K. 1981, ApJ, 246, L141

Jorstad, S. G., et al. 2005, AJ, 130, 1418

Kellermann, K. I., et al. 2004, ApJ, 609, 539

Lister, M. L., \& Homan, D. C. 2005, AJ, 130, 1389

Lister, M. L., Tingay, S. J., Murphy, D. W., Piner, B. G., Jones, D. L., \& Preston, R. A. 2001, ApJ, 554, 948
Miller, H. R., French, H. B., \& Hawley, S. A. 1978, ApJ, 219, L85

Mutel, R. L., Phillips, R. B., Su, B., \& Bucciferro, R. R. 1990, ApJ, 352, 81

Shepherd, M. C. 1997, in ASP Conf. Ser. 125, Astronomical Data Analysis Software and Systems VI, ed. G. Hunt \& H. E. Payne (San Francisco, CA: ASP), 77

Stirling, A. M., et al. 2003, MNRAS, 341, 405

Tateyama, C. E., \& Kingham, K. A. 2004, ApJ, 608, 149

Tateyama, C. E., Kingham, K. A., Kaufmann, P., Piner, B. G., de Lucena, A. M. P., \& Botti, L. C. L. 1998, ApJ, 500, 810

Villata, M., et al. 2004, A\&A, 424, 497 